\title{
A NOTE ON A CELLULARITY THEOREM BY DOYLE
}

D. G. STEWART ${ }^{1}$

A continuum $K$ in $S^{n}$ is cellular if $S^{n} \backslash K$ is topologically $E^{n}$. In [2] Doyle gave a sufficient condition for cellularity of an arc in $S^{n}$. In this paper we show that this condition is sufficient for cellularity of dendrites and can be used to get a condition for cellularity of unions of dendrites with cellular sets in $S^{n}$.

A continuum $K$ in $S^{n}$ is said to be $\mathcal{P}$-shrinkable if there is a point $q$ of $K$ and for each open set $U$ containing $q$ there is a closed $n$-cell $C \subset U$ such that $q$ lies in Int $C$ and $K$ meets $\mathrm{Bd} C$ in exactly one point. If $y$ is a point of $K$ different from $q$, we will say that $K$ is $\rho$ shrinkable towards $y$. The proofs of the following lemma and theorem are identical to those given by Doyle for Lemma 1 and Theorem 1 in [2] and, therefore, will not be given here.

Lemma 1. Let $C$ be a closed $n$-cell and $N$ a continuum which lies in Int $C$ except for a point $x$ of $N$ which lies on $\mathrm{Bd} C$. Then there is a pseudo-isotopy of $C$ onto $C$ which is fixed on $\mathrm{Bd} C$ and which carries $N$ onto $x$.

Theorem 1. Let $K$ be a continuum in $S^{n}$ which contains a cellular subset $L$. Suppose that each subcontinuum $K^{\prime}$ of $K$ which contains $L$ as a proper subcontinuum is P-shrinkable. Then, $K$ is cellular.

Corollary 1. If each subcontinuum of a dendrite in $S^{n}$ is $\beta$ shrinkable, then the dendrite, as well as each of its subcontinua, is cellular.

Corollary 2. Suppose $K$ is a dendrite, $y \in K$, and $L$ is a cellular set in $S^{n}$. Suppose also that $K \cap L=\{y\}$ and that each subcontinuum of $K$ containing $y$ is $P$-shrinkable towards $y$. Then, $K \cup L$ is cellular.

It is worthwhile to point out here that it is not enough to require that each arc of a dendrite be $P$-shrinkable in order to get cellularity of the dendrite. If we consider a dendrite on the Alexander horned sphere whose end points are the "bad" points of the horned sphere, we get a noncellular dendrite, each of whose arcs is tame and, therefore, $P$-shrinkable. If the dendrite has only a countable number of end points, then it would be sufficient to have each arc $P$-shrinkable.

Using the concept of being $P$-shrinkable, we get a local condition which gives cellularity. This condition, however, is not necessary.

Received by the editors August 19, 1966.

1 This work was supported by NSF Grant GP 5721. 
There are cellular arcs in $S^{3}$ which pierce no disk. Consider any arc of the simple closed curve described in [1]. Each subarc of such an arc would fail to be $P$-shrinkable.

To see that an arc of this simple closed curve is cellular, let $\left\{T_{i}\right\}_{i=1}^{\infty}$ be the nested sequence of solid tori which has the simple closed curve as its intersection. From $T_{1}$ remove a thickened disk $C$ such that $\mathrm{Cl}\left(T_{1} \backslash C\right)=C_{1}$ is a 3 -cell. Let $C_{2}$ be the 3 -cell of $T_{2} \cap C_{1}$ of largest diameter. In general let $C_{n+1}$ be the 3-cell of $T_{n+1} \cap C_{n}$ of largest diameter. Let $\alpha=\bigcap_{i=1}^{\infty} C_{i}$. Since each $C_{i}$ is tame, $\alpha$ is cellular.

\section{REFERENCES}

1. R. H. Bing, A simple closed curve that pierces no disk, J. Math. Pures Appl. 35 (1956), 337-343.

2. P. H. Doyle, $A$ sufficient condition that an arc in $S^{n}$ be cellular, Pacific J. Math. 14 (1964), 501-503.

Arizona State University 